\title{
Performance of Cognitive Hybrid Automatic Repeat reQuest: Stop-and-Wait
}

\author{
Ateeq Ur Rehman, Lie-Liang Yang, and Lajos Hanzo \\ School of Electronics and Computer Science, University of Southampton, SO17 1BJ, UK. \\ (E-mail: aur1g12, 1ly, lh@ecs.soton.ac.uk, http://www-mobile.ecs.soton.ac.uk)
}

\begin{abstract}
Detecting spectrum holes and efficiently accessing them are the two basic functions that enable a cognitive radio (CR) to make use of the licensed spectrums of a primary radio (PR). In this paper, we consider a CR scheme, which opportunistically accesses a PR channel for communication between a pair of nodes based on the stop-and-wait hybrid automatic repeat request (SW-HARQ). Hence, it is referred to as the cognitive SW-HARQ (CSW-HARQ) arrangement. In our CSW-HARQ system, the PR channel is modelled as a two-state Markov chain having 'On' and 'Off' states. The CR may only access the PR channel in its 'Off' state. In this paper, we analyze both the throughput and delay performance of the CSW-HARQ system, for which a range of closed-form formulas are derived that are also validated by simulation results. Our performance results show that both the activities of $P R$ users and the reliability of the CR channel have a substantial impact on the achievable performance of the CR system.
\end{abstract}

\section{INTRODUCTION}

Recent studies conducted both by the Federal Communication Commission (FCC) in the USA and by the European Telecommunications Standards Institute (ETSI) in Europe reveal that under the conventional static spectrum allocation policy substantial segments of the earmarked electromagnetic spectrum are heavily under-utilized [1]-[4]. As a result, the earmarked bandwidth cannot be exploited, whilst the readily available spectrum remains insufficient for innovative new wireless applications [3], [5]. This inefficient spectrum exploitation motivates the concept of dynamic spectrum access, which allows cognitive radio users (CRUs) to access and utilize the unoccupied spectrum holes, which have traditionally been exclusively assigned to primary radio users (PRUs) [5], [6].

The concept of CR was introduced by Mitola [7], which emerged as a promising paradigm for addressing the problem of spectrum scarcity. A CR is capable of detecting the unoccupied portions of the licensed spectrum and of efficiently accessing them for its own data transmission without affecting the legal rights of PRUs [8], [9]. Moreover, the $\mathrm{CR}$ is required to vacate the licensed spectrum as soon as the PRUs wish to access them. In order to improve the exploitation of the licensed spectrum, the regulatory bodies officially allow CRs to access and to opportunistically exploit the PR spectrum. As a result, the first CR based standard, namely IEEE 802.22, was ratified for wireless regional area networks (WRAN) [10].

In this paper, we propose a cognitive stop-and-wait hybrid automatic repeat request (CSW-HARQ) scheme. We focus our attention on opportunistic spectrum access in CR systems, where a CRU senses and occupies a PR channel for its own transmission, provided that the PR channel is free from PRUs. In order to analyze and detect the activity of PRUs on a channel, a number of sensing techniques were proposed in the literature [5], [11]-[14]. Specifically, in our studies, the activity of a PR channel is modelled using a two-state Markov chain [15], with the two states representing the 'On' and 'Off' status of PRUs. If the PR channel is deemed to be in its 'Off' state, the CR accesses the channel for its own transmission. Otherwise, it waits until the next time-slot and senses again. Corresponding to this scenario, a timeslot (TS) of duration $T$ is divided in the sensing epoch $\left(T_{s}\right)$ and the transmission epoch $\left(T_{d}\right)$. The sensing epoch $T_{s}$ is used for detecting spectrum holes, while the transmission epoch $T_{d}=T-T_{s}$ is used for data transmission. In our CR system, the data transmission relies on the principles of classic stop-and-wait hybrid automatic repeat request (SW-HARQ) [16]-[18].

Automatic Repeat reQuest (ARQ) constitutes an efficient technique of reliable data transmission over noisy channels. The concept of ARQ was originally introduced by Chang [19], which was then classified into three popular ARQ protocols: Stop-and-Wait ARQ (SWARQ), Go-Back-N ARQ (GBN-ARQ) and Selective-repeat ARQ (SRARQ) [16]-[18]. The principle of ARQ is appealingly simple. After transmitting a packet, if the original transmitter node fails to receive a positive acknowledgement within the defined time duration or if it receives a negative acknowledgement, the packet is retransmitted. The ARQ protocols are capable of achieving reliable data transmission, provided that the channel-induced error rate remains moderate. However, beyond a certain error rate both the throughput and the delay may become inadequate. Hence, for the sake of enhancing the performance, hybrid Forward Error Correction (FEC) and ARQ (HARQ) schemes [20], [21] may be employed. In addition to detecting errors, in HARQ, the FEC scheme also has the capability of correcting a number of errors and the ARQ mechanism is activated for the retransmission of a packet, when residual errors are detected after FEC decoding. As a benefit, HARQ schemes are typically capable of providing a better throughput/delay performance than the corresponding ARQ schemes. Hence, they have been widely used in wireless communication systems [22], [23].

In this paper, our proposed CSW-HARQ scheme intrinsically amalgamates CR with the classic SW-HARQ regime. It first senses a PR channel and, if finds it free, it accesses the channel and transmits packets using our CSW-HARQ scheme. In this paper, Reed-Solomon (RS) codes are used for error correction and detection [24]-[26]. We investigate both by analysis and simulations both the achievable throughput and delay performance of CSW-HARQ. We will demonstrate that both the behaviour of the PR users as well as the quality of the CR channel have a substantial impact on the achievable throughput and delay performance of the CR system.

The rest of this paper is organized as follows. The system considered is described in Section II. The principle of our CSW-HARQ scheme is outlined in Section II-B. Section III analyses the attainable throughput and delay performance, followed by the performance results given in Section IV. Finally, our conclusions are presented in Section V.

\section{SYSTEM MODEL}

\section{A. PR System and Assumptions}

Let us consider a communication channel, which allows a PR system to transmit packets of duration $T_{p}$. We assume that the PR system uses the channel according to a discrete-time Markov chain having two states, namely 'On' and 'Off', as shown in Fig. 1. The transition probabilities from the 'On/Off' state to the 'Off/On' state are expressed as $\alpha$ and $\beta$, respectively. Let us assume that the probabilities of the PR system being in the 'On' and 'Off' states are $P_{\text {on }}$ and $P_{o f f}=1-P_{o n}$, which are the probability of the channel being occupied by the PR and that of the channel being free from the PR. 


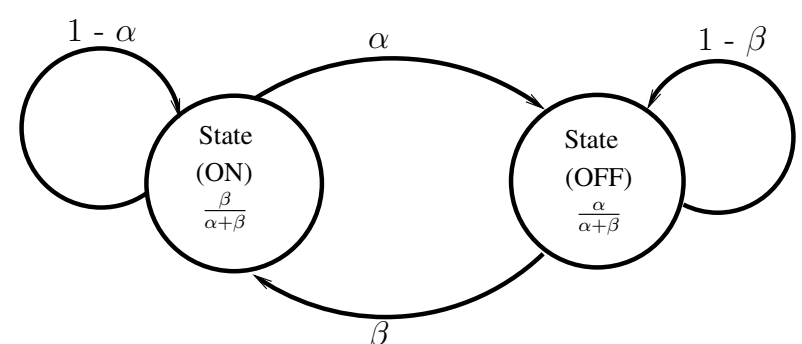

Fig. 1. Two-state discrete-time Markov chain model of the PR system.

Then, provided that the Markov chain is in its steady state, we have [16]

$$
P_{\text {on }} \alpha=P_{\text {off }} \beta \text {, }
$$

which gives

$$
P_{o n}=\frac{\beta}{\alpha+\beta}, \quad P_{o f f}=\frac{\alpha}{\alpha+\beta} .
$$

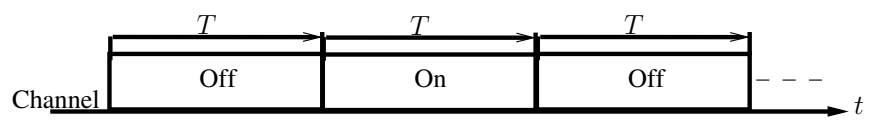

(a) Pattern of channel usage by a PR

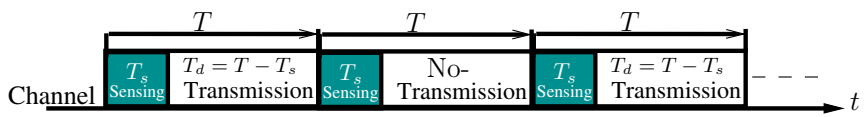

(b) Pattern of channel usage by a CR

Fig. 2. Time-slot structure of a CR system having a sensing duration $T_{s}$ and a transmission duration $T_{d}$, when the total duration of a time-slot is $T$ [27].

We assume that in the PR system the time is divided into TSs of duration $T$ and that the PR users always activate the channel at the start of a TS, as shown in Fig. 2(a). When the channel is 'On', one of the PR users occupies the channel and transmits a few packets. By contrast, if the channel is detected to be in the 'Off' state, a CR user accesses the channel and transmits its packets. For simplicity, we assume that the activities of the PR users within different TSs are independent of each other.

\section{B. Cognitive Stop-and-Wait Hybrid Automatic Repeat Request}

We assume that the CR system works in the high SNR region of the PR system, and it is capable of reliably sensing the 'On/Off' state of the PR channel, without false-alarm and also without miss-detection. The time-slots in the CR system are structured in a form as shown Fig. 2(b). Within a time-slot of $T$ seconds, the CR transmitter dedicates $T_{s}$ seconds at the start to channel sensing and then uses the remaining $T_{d}=T-T_{s}$ seconds to transmit data, provided that the PR channel is found in the 'Off' state. Otherwise, the CR transmitter waits and senses again the PR channel at the commencement of the next time-slot. In the CR system, the data to be transmitted are assumed to be encoded by a RS code $R S\left(N_{d}, K_{d}\right)$ [17], where $K_{d}$ and $N_{d}$ represent the number of information and coded symbols, respectively. We assume for simplicity that a CR packet is encoded by a single RS codeword, which is transmitted during $T_{p}<T_{d}$ seconds. We assume that the RS code is capable of correcting $t=\left(N_{d}+K_{d}\right) / 2$ symbol errors and that it has a perfectly reliable error-detection probability of one, as and when there are uncorrectable errors.

Given the above assumptions, the data are transmitted between a pair of CR users over the PR channel based on the principles of our CSW-HARQ scheme, which is characterized in Fig. 3 as well as in Algorithm 1 and detailed below.

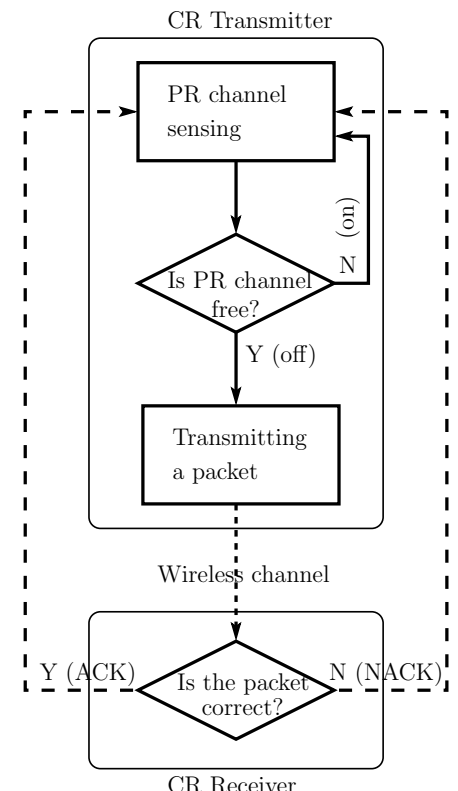

Fig. 3. Flow chart showing the operations of the proposed CSW-HARQ scheme.

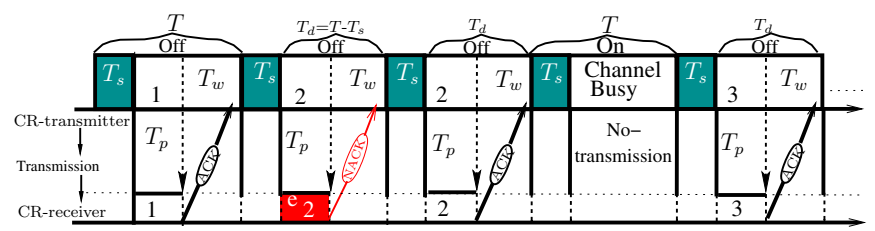

Fig. 4. The transmission flow of the proposed CSW-HARQ scheme. The total duration of each time-slot is $T=T_{s}+T_{d}$, where $T_{d}$ consists of a packet's transmission duration and its waiting epoch $T_{w}$.

\section{Operation of the CR Transmitter}

In the classic SW-HARQ, the transmitter sends a single packet in a TS and then waits for its feedback, which is expected to be received within a specified round-trip time (RTT). By contrast, in the CSWHARQ, the CR transmitter has to sense the PR channel before the transmission or retransmission of a packet. If the PR channel is deemed to be in the 'Off' state, the CR transmitter sends a packet. Otherwise, it has to wait and sense again. Again, the CSW-HARQ procedure is portrayed in both the Algorithm 1 as well as in Fig. 3. Similar to the classic SW-HARQ scheme, the CR transmitter has a buffer of size one, which is updated based on the feedback flag of each packet.

We assume that all packets are of the same length and that the CR

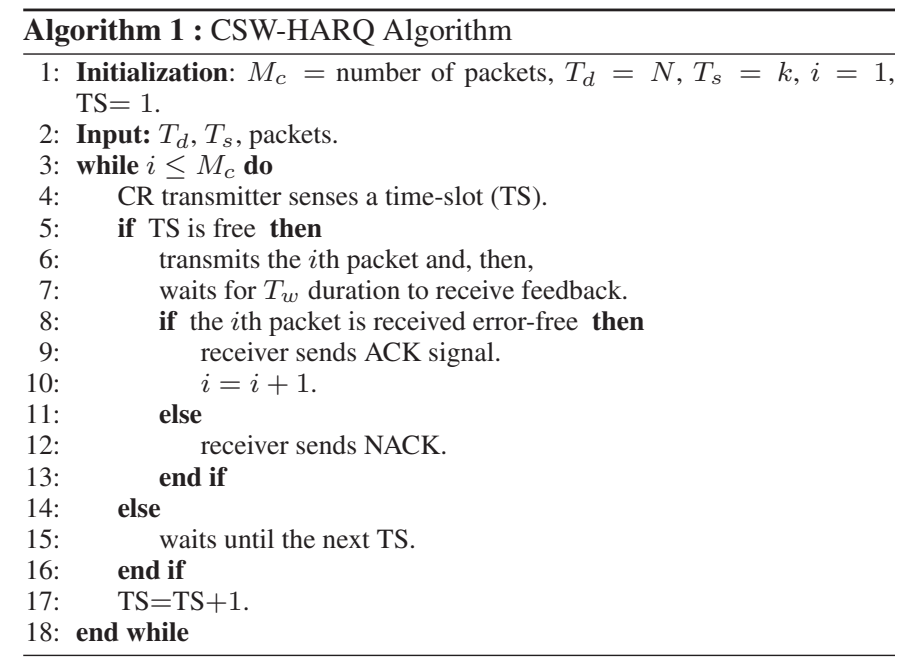


transmitter is always ready to transmit these packets in free TSs. As shown in Fig. 4, each CR packet consists of a RS coded codeword, which is transmitted within the duration of $T_{p}$ seconds. After transmission of a packet, the CR transmitter waits for $T_{w}$ duration in order to receive its feedback. We assume that the RTT is $T_{d}$, which is the time interval between the transmission of a packet and the instant, when its feedback is received. Therefore, in our CSW-HARQ scheme, the feedback of each packet is received within the RTT of duration $T_{d}$, as shown in Fig. 4.

Similar to the principle of the classic SW-HARQ schemes [16][19], in our proposed CSW-HARQ arrangement, the CR transmitter is not allowed to transmit or retransmit its packets, while it is waiting for the feedback flag of the transmitted packet. Hence, if a positive feedback (i.e., ACK) of a packet is received by the original transmitter node within the RTT, then this packet is deleted from the transmitter buffer and a new packet is transmitted in the next free TS. However, if a negative feedback (i.e., NACK) is received, then the transmitter retransmits the erroneous packet in the next free TS.

\section{Operation of the CR Receiver}

When the CR receiver receives a packet from the CR transmitter, it invokes RS error-correction/detection and then generates a feedback flag accordingly. Specifically, if a packet is correctly detected by the RS decoder, an ACK signal is fed back to the CR transmitter, otherwise, a NACK signal is sent to the CR transmitter for requesting retransmission. In this paper, we assume that the feedback channel is perfect, and the receiver has a buffer of size one, which is updated only when a packet is correctly received [16]-[18].

For example, as shown in Fig. 4, packet 1 is correctly received by the CR receiver. Therefore, an ACK signal is fed back for this packet and the sequence index of the receiver buffer is increased by one. After receiving the feedback of the corresponding packet, the CR transmitter senses the next free TS and transmits a new packet, i.e. packet 2, as seen in Fig. 4. However, this packet is seen to be detected in error after RS decoding. Therefore, the CR receiver uses a NACK signal to ask the CR transmitter for a retransmission. After the reception of the NACK signal, the CR transmitter retransmits packet 2 in the next TS, since the PR channel is detected to be free in this TS. By contrast, as shown in Fig. 4, if the PR channel is found to be in the 'On' state (Busy), the CR transmitter stops its transmissions and it waits until the next TS and senses again. The above process continues, until all packets are successfully received by the $\mathrm{CR}$ receiver.

\section{Performance AnAlysis of CSW-HARQ}

In this section, we analyze both the attainable throughput and the average packet delay. In our analysis of the average packet delay, the total time spanning from the start of the PR channel's sensing to the successful transmission of all the packets is taken into account, i.e. both the free and busy TSs are counted right from the start of the CR system's activation for transmission of a block of packets. By contrast, in our simulations reported in Section IV, we additionally consider another type of delay, referred to as the end-to-end packet delay, which is the time from the start of transmitting a packet to the instant that the packet is confirmed to be successfully received. In our analysis, we assume that the time required for preparing packets and the ACK/NACK feedback duration can be ignored. Furthermore, we assume that the errors generated by the undetectable errors of the RS code can be ignored.

\section{A. Delay}

In contrast to the classic SW-HARQ, where the delay is only imposed by the channel introduced errors, in our CSW-HARQ, the delay includes both that caused by channel errors and by the lack of free channels for the $\mathrm{CR}$ transmitter to use. Let $T_{D P}$ and $T_{D}$ represent the delay imposed by the PR channel's busy state and the delay incurred by the $\mathrm{CR}$ to successfully transmit a packet to the $\mathrm{CR}$ receiver, respectively. Then, given the probability $P_{\text {on }}$ of the PR system, where $P_{\text {on }}$ is defined in (2), the average delay $T_{D P}$ for the CR system to find a free channel can be formulated as

$$
\begin{aligned}
T_{D P} & =E\left[T_{D P}(i)\right] \\
& =E[(i-1) T] \\
& =\sum_{i=1}^{\infty}(i-1) T P_{o n}^{i-1}\left(1-P_{o n}\right) \\
& =\frac{P_{\text {on }} T}{1-P_{\text {on }}}
\end{aligned}
$$

where $T_{D P}(i)$ denotes the delay, when the CR transmitter detects that the $i$ th TS is free for its use, while the previous $(i-1)$ TSs are busy, which gives a delay of $(i-1) T$. Furthermore, when substituting $P_{\text {on }}=$ $\beta /(\alpha+\beta)$ from (2) into the above equation, we arrive at

$$
T_{D P}=\frac{\beta T}{\alpha} .
$$

The average packet delay $T_{D}$ can be expressed as

$$
\begin{aligned}
T_{D} & =E\left[T_{D}(i)\right] \\
& =E\left[\left(i\left(T_{D P}+T\right)\right],\right.
\end{aligned}
$$

where $T_{D}(i)$ denotes the average delay, when the CR transmitter uses in total $i$ transmissions to successfully send a packet to the CR receiver. Explicitly, we have $T_{D}(i)=i\left(T_{D P}+T\right)$, since for every $\mathrm{CR}$ transmission, the $\mathrm{CR}$ transmitter requires in average duration of $T_{D P}$ to find a free TS and then uses the free TS to transmit a packet. Let us denote the packet error probability after RS decoding by $P_{F}$. Then, we have

$$
\begin{aligned}
T_{D} & =\sum_{i=1}^{\infty} i\left(T_{D P}+T\right) P_{F}^{i-1}\left(1-P_{F}\right) \\
& =\frac{(\alpha+\beta) T}{\alpha} \sum_{i=1}^{\infty} i P_{F}^{i-1}\left(1-P_{F}\right) \\
& =\left(1+\frac{\beta}{\alpha}\right) \frac{T}{1-P_{F}} \text { (seconds). }
\end{aligned}
$$

Eq.(6) shows that when the PR channel becomes busier, resulting in an increase of $\beta / \alpha$, and/or when the CR channel becomes less reliable reflected by the increase of $P_{F}$, the average packet delay increases.

\section{B. End-to-End Throughput}

The throughput of our CSW-HARQ scheme can be defined as the total number of packets successfully delivered from the CR transmitter to the CR receiver per TS. Explicitly, the event of successfully delivering a packet depends on two events: (a) the CR transmitter successfully detects a free TS and (b) the CR transmitter uses the free TS to successfully deliver a packet. Let us express the probability $P_{S}(i)$ that a packet is successfully delivered by the CR transmitter to the CR receiver using $i$ TSs. This implies that the packet is only successfully delivered within the $i$ th TS. For the other $(i-1)$ TSs, the PR channel might be occupied by the PR user or the packet was received in error, hence requires retransmission. Therefore, the probability $P_{S}(i)$ can be expressed as

$$
P_{S}(i)=\sum_{j=1}^{i} P_{o f f}(j \mid i) P_{S}(j \mid i),
$$

where $P_{\text {off }}(j \mid i)$ denotes the probability that the PR channel is free in $j$ out of the $i$ TSs, while $P_{S}(j \mid i)$ denotes the probability that the CR 
transmitter uses $j$ TSs to transmit the packet successfully to the CR receiver. Hence, we have

$$
\begin{aligned}
P_{S}(i) & =\sum_{j=1}^{i}\left(\begin{array}{l}
i \\
j
\end{array}\right) P_{o f f}^{j} P_{o n}^{i-j} P_{F}^{j-1}\left(1-P_{F}\right) \\
& =\sum_{j=1}^{i}\left(\begin{array}{l}
i \\
j
\end{array}\right)\left(\frac{\alpha}{\alpha+\beta}\right)^{j}\left(\frac{\beta}{\alpha+\beta}\right)^{i-j} P_{F}^{j-1}\left(1-P_{F}\right) .
\end{aligned}
$$

Based on the above analysis, the total number of packets successfully delivered by the $\mathrm{CR}$ transmitter to the CR receiver per TS, which is the normalized throughput, can be expressed as

$$
\begin{aligned}
R_{s}= & \sum_{i=1}^{\infty} \frac{1}{i} \times P_{S}(i) \\
= & \sum_{i=1}^{\infty} \sum_{j=1}^{i} \frac{1}{i}\left(\begin{array}{l}
i \\
j
\end{array}\right)\left(\frac{\alpha}{\alpha+\beta}\right)^{j}\left(\frac{\beta}{\alpha+\beta}\right)^{i-j} \\
& \times P_{F}^{j-1}\left(1-P_{F}\right) \text { (packets per TS). }
\end{aligned}
$$

Furthermore, if we express the throughput in terms of packets per $T_{p}$ (packet duration), we have

$$
R_{s}^{\prime}=\frac{T_{p}}{T} \times R_{s}=\frac{T_{p}}{T_{s}+T_{d}} \times R_{s} \quad\left(\text { packets per } T_{p}\right) .
$$

\section{Performance Results}

In this section, both the throughput and delay performance of the CSW- HARQ scheme are characterized in terms of two factors: 1) the PR channel's relative occupancy reflected by $P_{o n}$, and 2) the CR channel's reliability quantified by $P_{F}$.

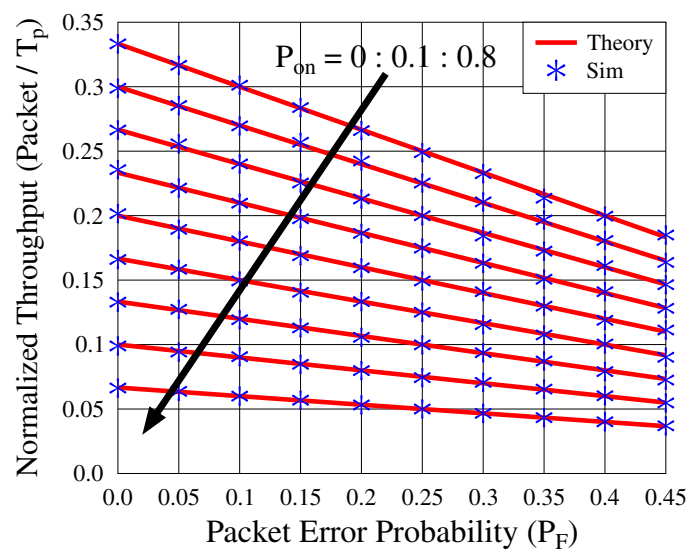

Fig. 5. Throughput performance of our CSW-HARQ scheme versus the packet error probability $\left(P_{F}\right)$ for various probabilities of the channel being busy $\left(P_{o n}\right)$, when we have $T_{s}=1 T_{p}$ and $T_{d}=2 T_{p}$.

Fig. 5 depicts the throughput of the CSW-HARQ scheme versus the packet error probability $\left(P_{F}\right)$ for various PR channel occupancy probabilities of $P_{o n}$. In our simulations, the throughput $\left(R_{S}^{\prime}\right)$ is calculated according to:

$$
R_{S}^{\prime}=\frac{N_{s}}{N_{t}} \times \frac{T_{p}}{T_{s}+T_{d}}\left(\text { packets per } T_{p}\right),
$$

where $N_{t}$ represents the total number of TSs used for the successful transmission of $N_{s}$ packets by the CR. For a given $P_{o n}$, it is observed from Fig. 5 that the throughput of CSW-HARQ is at its maximum, when $P_{F}=0$. However, an increase of $P_{F}$ degrades the reliability of the channel, resulting in an increased number of retransmitted packets and hence in a reduced throughput. Additionally, for a given $P_{F}$, the throughput of the CSW-HARQ scheme is maximum, when the PR channel is always 'Off', corresponding to $P_{o n}=0$. However, when the probability of the PR channel's occupancy increases, i.e. if the value of $P_{\text {on }}$ increases from 0 to 0.8 , the throughput of the CR system is reduced, since the $\mathrm{CR}$ system has less opportunities for transmission over the PR channel. Additionally, we can see that the analytical results evaluated from Equation (9) agree well with the simulation results.

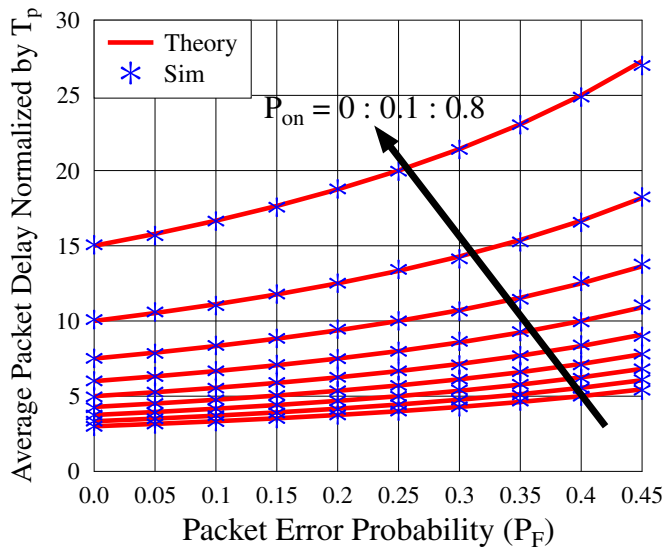

Fig. 6. Average packet delay of the CSW-HARQ system versus the packet error probability, when we have $T_{s}=1 T_{p}$ and $T_{d}=2 T_{p}$.

Fig. 6 portrays the average packet delay of the CSW-HARQ scheme. In our simulations, the average packet delay $\left(T_{D S}\right)$ is obtained by:

$$
T_{D S}=\frac{N_{t} \times\left(T_{s}+T_{d}\right)}{N_{s}} \text { (seconds). }
$$

Fig. 6 shows that the average packet delay of the CSW-HARQ scheme is at its minimum, when the CR channel is error free, i.e. when $P_{F}=0$, and the probability of the PR channel occupancy is zero, i.e. $P_{o n}=0$. The average delay increases with the degradation of the CR channel. The average delay also increases, when the channel occupancy of the PR users increases. Again, the comparison between analytical and simulation results shown in Fig. 6 demonstrates that Eq. (6) is accurate.

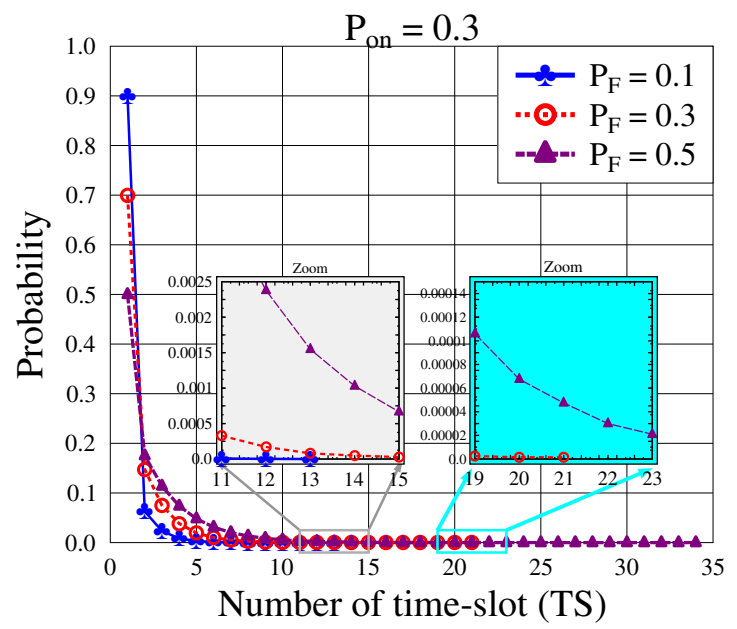

Fig. 7. Probability of a specific end-to-end packet delay of the CSW-HARQ system expressed as a function of the number of TSs for various values of $P_{F}$, $P_{o n}=0.3, T_{s}=1 T_{p}$ and $T_{d}=2 T_{p}$.

The probability mass function (PMF) of the end-to-end packet delay generated by the CSW-HARQ is shown in Fig. 7 in terms of the number of TSs. As mentioned in the first paragraph of Section III, the end-to-end packet delay represents the time duration from a packet's first transmission attempting until the confirmation of its successful reception. This includes both the 'On' TSs and 'Off' TSs during the transmission of a packet. Let $\boldsymbol{d}$ be a vector having the $k$ th element $\boldsymbol{d}(k)$ storing the recorded end-to-end delay of the $k$ th packet. Furthermore, let $N_{s}$ be the total number of packets successfully transmitted. Our 
PMF seen in Fig. 7 for the end-to-end packet delay is formulated as

$$
\boldsymbol{P}_{d}(i)=\frac{\sum_{k=1}^{N_{s}} \delta(d(k)-i)}{N_{s}}, 1 \leq i \leq \max (\boldsymbol{d}) .
$$

We can observe from Fig. 7 that the PMF for $P_{F}=0.1$ has a maximum value of 0.9 , meaning that $90 \%$ of the packets are successfully transmitted with a delay of one TS. Furthermore, an additional $6 \%$ of the packets are successfully received using two TSs. As shown in Fig 7, when $P_{F}$ increases, the PMF curves shift towards the righthand side, implying an increase of the end-to-end packet delay.

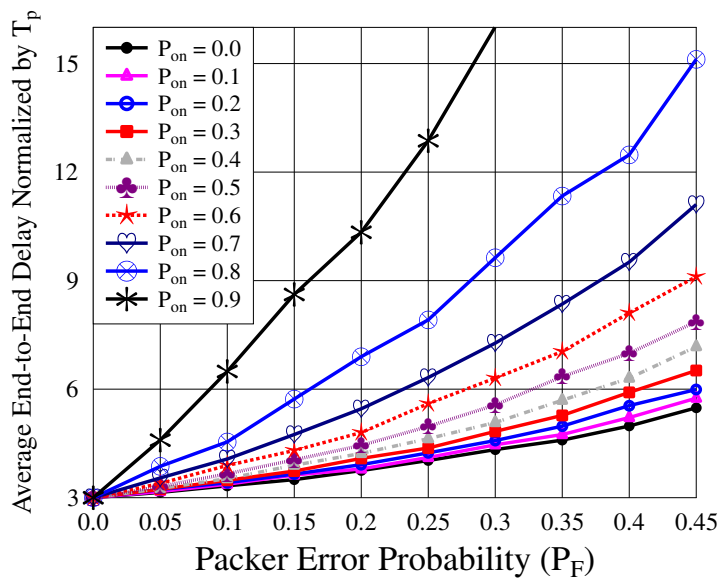

Fig. 8. Average end-to-end packet delay of the CSW-HARQ scheme versus $P_{F}$ for the various values of $P_{o n}$, when we have $T_{s}=1 T_{p}$ and $T_{d}=2 T_{p}$.

Continuing on from Fig. 7, in Fig. 8, we investigate the average endto-end packet delay, which is evaluated from

$$
\tau=\sum_{i=1}^{\max (\boldsymbol{d})} \boldsymbol{P}_{d}(i) \times i\left(T_{s}+T_{d}\right) \text { (seconds). }
$$

It can be observed from Fig. 8 that the average end-to-end packet delay increases, as $P_{F}$ and/or $P_{o n}$ increase, which indicates the same trend as that shown in Fig. 6. However, when making a close comparison between Fig. 6 and Fig. 8, we find that the average end-to-end packet delay is lower than the corresponding average packet delay. This is because the packet delay includes not only the end-to-end packet delay, but also the busy TSs before sending a new packet, which are not accounted for the end-to-end packet delay. This also explains that at a given $P_{F}$, the average end-to-end packet delay is much lower than the corresponding average packet delay, when $P_{o n}$ is relatively high, hence resulting in numerous busy TSs.

\section{Conclusions}

In this paper, we have proposed a CSW-HARQ transmission scheme for a CR to access a PR channel. Both the throughput and delay performance of the proposed CSW-HARQ scheme have been investigated both by analysis and simulations. A range of formulas have been derived and the performance of the CSW-HARQ systems has been compared from different perspectives. Our simulation results demonstrates that the analytical formulas are accurate. Based on our performance results, we can conclude that the both achievable throughput and delay performance of the CSW-HARQ is substantially affected by both the activities of the PR and by the reliability of the CR channels. When the PR channel is busy, the CSW-HARQ's throughput might become very low and its packet delay might become excessive, even when the CR's communication channel is reliable. Our future research on this topic will be considering realistic imperfect sensing scenarios as well as other types of ARQ schemes.

\section{REFERENCES}

[1] F. Communications Commission, "Spectrum policy task force report Technical Report ET Docket No. 02-155," Nov 2002.

[2] F. communications Commission, "Notice of proposed rule making and order ET Docket No 03-222," December 2003.

[3] G. Staple and K. Werbach, "The end of spectrum scarcity," IEEE, Spectrum, vol. 41, no. 3, pp. 48-52, 2004

[4] M. A. McHenry, P. A. Tenhula, D. McCloskey, D. A. Roberson, and C. S. Hood, "Chicago spectrum occupancy measurements \& analysis and a long-term studies proposal," in Proceedings of the First International Workshop on Technology and Policy for Accessing Spectrum, ser. TAPAS '06. New York, NY, USA: ACM, 2006.

[5] E. Hossain, D. Niyato, and Z. Han, Dynamic spectrum access and management in cognitive radio networks. Cambridge University Press, 2009.

[6] Q. Zhao and B. Sadler, "A survey of dynamic spectrum access," IEEE Signal Processing Magazine, vol. 24, no. 3, pp. 79-89, May 2007.

[7] J. Mitola and J. Maguire, G.Q., "Cognitive radio: making software radios more personal," IEEE Personal Communications, vol. 6, no. 4, pp. 13-18, Aug 1999.

[8] S. Haykin, "Cognitive radio: brain-empowered wireless communications," IEEE Journal on Selected Areas in Communications, vol. 23, no. 2, pp. 201-220, Feb 2005.

[9] J. Hu, L. liang Yang, and L. Hanzo, "Maximum average service rate and optimal queue scheduling of delay-constrained hybrid cognitive radio in nakagami fading channels," IEEE Transactions on Vehicular Technology, vol. 62, no. 5, pp. 2220-2229, Jun 2013.

[10] "IEEE Recommended Practice for Information Technology - Telecommunications and information exchange between systems Wireless Regional Area Networks (WRAN) - Specific requirements - Part 22.2: Installation and Deployment of IEEE 802.22 Systems," IEEE Std 802.22.2-2012, pp. $1-44$, Sept 2012.

[11] O. Akan, O. Karli, and O. Ergul, "Cognitive radio sensor networks," IEEE Network, vol. 23, no. 4, pp. $34-40$, July-August 2009.

[12] T. Yucek and H. Arslan, "A survey of spectrum sensing algorithms for cognitive radio applications," IEEE Communications Surveys Tutorials, vol. 11, no. 1, pp. 116-130, Quarter 2009.

[13] R. Umar and A. U. Sheikh, "A comparative study of spectrum awareness techniques for cognitive radio oriented wireless networks," Physical Communication, vol. 9, no. 0, pp. 148 - 170, 2013.

[14] J. Ma, G. Li, and B. H. Juang, "Signal processing in cognitive radio," Proceedings of the IEEE, vol. 97, no. 5, pp. 805-823, May 2009.

[15] G. Ozcan and M. Gursoy, "Throughput of cognitive radio systems with finite blocklength codes," IEEE Journal on Selected Areas in Communications, vol. 31, no. 11, pp. 2541-2554, November 2013.

[16] D. Bertsekas and R. Gallagher, Data Networks, 2nd ed. Englewood Cliffs, NJ: Prentice Hall, 1991.

[17] S. Lin and D. J. C. Jr., Error Control Coding: Fundamentals and Applications, 2nd ed. Upper Saddle River, NJ: Prentice-Hall, 1999.

[18] A. Leon-Garcia and I. Widjaja, Communication Networks. McGraw-Hill Education, 2004.

[19] S. Chang, "Theory of information feedback systems," IRE Transactions on Information Theory, vol. 2, no. 3, pp. 29-40, September 1956.

[20] J. Wozencraft and M. Horstein, "Digitalised communication over twoway channels," in Fourth London Symp. Inform. Theory, London, UK, Sept 1960.

[21] J. M. Wozencraft and M. Horstein, "Coding for two-way channels," 1961.

[22] "IEEE standard for local and metropolitan area networks part 16: Physical and medium access control layers for combined fixed and mobile operation in licensed bands and corrigendum 1," IEEE Std 802.16e-2005 and IEEE Std 802.16-2004/Cor 1-2005 (Amendment and Corrigendum to IEEE Std 802.16-2004), pp. 1-822, Feb 2006.

[23] 3GPP TR 25.848 V4.0.0, "3rd Generation Partnership Project; Technical Specification Group Radio Access Network; Physical layer aspects of UTRA High Speed Downlink Packet Access (Release 4)," 2001.

[24] W. C. Ao and K. C. Chen, "End-to-end harq in cognitive radio networks," in Proc. IEEE Wireless Communications and Networking Conference (WCNC) Sydney, Australia, Apr. 2010, pp. 1-6.

[25] C. Lott, O. Milenkovic, and E. Soljanin, "Hybrid arq: Theory, state of the art and future directions," in IEEE Information Theory Workshop on Information Theory for Wireless Networks, 2007, pp. 1-5.

[26] G. Mitchell, "Investigation of hamming, reed-solomon, and turbo forward error correcting codes," DTIC Document, Tech. Rep., 2009.

[27] S. Stotas and A. Nallanathan, "Enhancing the capacity of spectrum sharing cognitive radio networks," IEEE Transactions on Vehicular Technology, vol. 60, no. 8, pp. 3768-3779, 2011. 